Published in final edited form as:

Am J Obstet Gynecol. 2018 August ; 219(2): 178.e1-178.e10. doi:10.1016/j.ajog.2018.05.034.

\title{
A prospective cohort study of meat and fish consumption and endometriosis risk
}

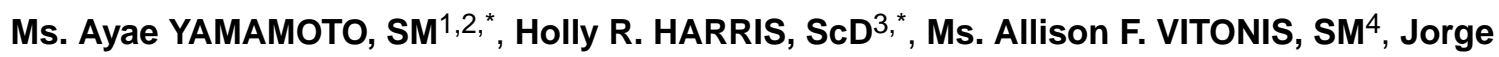 \\ E. CHAVARRO, MD, ScD ${ }^{1,5,6}$, and Stacey A. MISSMER, ScD ${ }^{1,7,8}$ \\ ${ }^{1}$ Department of Epidemiology, Harvard T.H. Chan School of Public Health, 677 Huntington \\ Avenue, Boston, Massachusetts, United States, 02115 \\ ${ }^{2}$ Department of Health Policy and Management, University of California-Los Angeles Field School \\ of Public Health, 650 Charles Young Drive South, Los Angeles, California, United States, 90095 \\ ${ }^{3}$ Program in Epidemiology, Division of Public Health Sciences, Fred Hutchinson Cancer Research \\ Center, 1100 Fairview Avenue North, M4-B859, Seattle, Washington, United States, 98109 \\ ${ }^{4}$ Obstetrics and Gynecology Epidemiology Center, Department of Obstetrics and Gynecology, \\ Brigham and Women's Hospital, 221 Longwood Avenue, Boston, Massachusetts, United States, \\ 02115
}

${ }^{5}$ Channing Division of Network Medicine, Department of Medicine, Brigham and Women's Hospital and Harvard Medical School, 181 Longwood Avenue Boston, Massachusetts, United States, 02115

${ }^{6}$ Department of Nutrition, Harvard T.H. Chan School of Public Health, 665 Huntington Avenue, Boston, Massachusetts, United States, 02115

${ }^{7}$ Division of Adolescent and Young Adult Medicine, Department of Medicine, Boston Children's Hospital and Harvard Medical School, 333 Longwood Avenue, Boston, Massachusetts, United States, 02115

${ }^{8}$ Department of Obstetrics, Gynecology, and Reproductive Biology, College of Human Medicine, Michigan State University, 15 Michigan Street NE, Grand Rapids, Michigan, United States, 49503

\section{Abstract}

Background-Only two case-control studies have examined the associations between consumption of meat products and endometriosis risk with inconsistent results. Consumption of animal products has the potential to influence endometriosis risk through effects on steroid hormones levels.

Corresponding author: Dr. Holly R. Harris, Program in Epidemiology, Division of Public Health Sciences, Fred Hutchinson Cancer Research Center, 1100 Fairview Avenue North, M4-859, Seattle, WA 98109-1024, Telephone: 206-667-2712, hharris@ fredhutch.org. ${ }^{*}$ Contributed equally as first authors to this work.

Conflict of Interest Disclosure Statement: The authors report no conflict of interest.

Publisher's Disclaimer: This is a PDF file of an unedited manuscript that has been accepted for publication. As a service to our customers we are providing this early version of the manuscript. The manuscript will undergo copyediting, typesetting, and review of the resulting proof before it is published in its final citable form. Please note that during the production process errors may be discovered which could affect the content, and all legal disclaimers that apply to the journal pertain. 
Objectives-To determine whether higher intake of red meat, poultry, fish, and seafood are associated with risk of laparoscopically-confirmed endometriosis

Study Design-81,908 participants of the prospective Nurses' Health Study II were followed from 1991-2013. Diet was assessed via food frequency questionnaire every 4 years. Cox proportional hazards models were used to calculate rate ratios (RR) and $95 \%$ confidence intervals (CIs).

Results-During 1,019,294 person-years of follow-up, 3,800 cases of incident laparoscopicallyconfirmed endometriosis were reported. Women consuming $>2$ servings/day of red meat/day had a $56 \%$ higher risk in endometriosis $\left(95 \% \mathrm{CI}=1.22-1.99 ; \mathrm{P}_{\text {trend }}<0.0001\right)$ compared to those consuming $\leq$ serving/week. This association was strongest for non-processed red meats $\left(\mathrm{RR}=1.57 ; 95 \% \mathrm{CI}=1.35-1.83\right.$ for $\geq 2$ servings/day versus $\leq$ servings/week; $\left.\mathrm{P}_{\text {trend }}<0.0001\right)$, particularly among women had not reported infertility ( p interaction $=0.0004)$. Women in the highest category of processed red meat intake also had a higher risk of endometriosis $(\mathrm{RR}=1.20 ; 95 \%$ $\mathrm{CI}=1.06-1.37$ for $\geq 5$ servings/week versus $<1$ serving/month; $\left.\mathrm{P}_{\text {trend }}=0.02\right)$. Intakes of poultry, fish, shellfish, and eggs were unrelated to endometriosis risk.

Conclusions-Our prospective analysis among premenopausal US nurses suggests that red meat consumption may be an important modifiable risk factor for endometriosis, particularly among women with endometriosis who had not reported infertility and thus were more likely to present with pain symptoms. Well-designed dietary intervention studies among women with endometriosis could help confirm this observation.

\section{Keywords}

endometriosis; diet; meat

\section{Introduction}

Endometriosis is a benign, estrogen-dependent, gynecologic condition with a prevalence of $\sim 10 \%$ in women of reproductive age ${ }^{1}$. It is characterized by the presence of endometrial tissue outside of the uterus, causing inflammation and leading to the formation of scars and adhesions. Endometriosis patients experience a variety of symptoms including chronic pelvic pain and infertility. Despite that it is the third leading cause of gynecologic hospitalizations in the US, its etiology is not fully understood ${ }^{2}$.

There has been an increased interest in the identification of modifiable risk factors for endometriosis, such as diet and exercise. Diet may influence endometriosis risk through its influence on steroidal hormones. For example, red meat has been shown to decrease sex hormone-binding globulin (SHBG) and increase estradiol concentrations ${ }^{3}$, while fish oil has been associated with lower circulating levels of series 2 prostaglandins and decreased inflammatory symptoms ${ }^{4}$, as well as a decrease in dysmenorrhea ${ }^{5}$. Estrogen up-regulates prostaglandin synthesis and evidence of positive feedback for local estrogen and prostaglandin may favor the inflammatory and proliferative characteristics of endometriosis ${ }^{6}$. Although there is extensive lay literature touting dietary changes to reduce endometriosis and symptoms, scientific literature in the field remains scant. An Italian case-control study reported that women with endometriosis had higher consumption of red meat and ham and 
lower intake of fish than women without endometriosis ${ }^{7}$. In contrast, a Washington state based case-control study reported no association between intake of red meat or seafood and endometriosis diagnosis ${ }^{8}$.

In this prospective study, we investigated the association between intake of red meat, poultry, fish, seafood, and nutrients concentrated in red meats (iron and heme iron) and risk of laparoscopically-confirmed endometriosis using data from the Nurses' Health Study II. We also examined whether these associations differed by fertility status.

\section{Materials and Methods}

\section{Study Population}

The Nurses' Health Study II (NHSII) prospective cohort consists of 116,429 female registered nurses who were ages $26-42$ at baseline. This cohort has been followed from 1989 when the baseline questionnaire regarding information on disease history, demographic, anthropometric, and lifestyle risk factors was completed and subsequently, biennial questionnaires have been administered. Implied consent was assumed upon completion and return of the questionnaires. This study was approved by the Institutional Review Board of Brigham and Women's Hospital. Follow-up for this analysis include questionnaire data beginning in 1991 when 97,807 NHSII participants completed the 1991 FFQ and concluded in 2013. Response rates have been approximately $\geq 0 \%$ for each questionnaire cycle. We restricted our analyses to premenopausal women with intact uteri, with no prior diagnosis of endometriosis, or cancer (with the exception of non-melanoma skin cancer) prior to June 1991. In addition we excluded women with implausible total energy intake ( $<800$ or $>4200 \mathrm{kcal} / \mathrm{day}$ ), or who left more than 70 food items blank on the $1991 \mathrm{FFQ}$. There were 81,908 participants remaining in the study after these exclusions.

\section{Case ascertainment}

Starting with the 1993 questionnaire, the nurses were asked if they have "ever had physiciandiagnosed endometriosis." If participants reported "yes", they were asked if it was confirmed by laparoscopy, which is considered the "gold standard" for endometriosis diagnosis. For each subsequent questionnaire cycle, participants were asked diagnosis of endometriosis in the preceding two years. From the 1766 incident cases that were identified from the initial questionnaire, a validation study was conducted the following year among 200 randomly selected cases ${ }^{9}$. A supplemental questionnaire was mailed to these participants requesting for permission to review their clinical and surgical records. In $96.2 \%$ of the 105 cases in which laparoscopic confirmation was reported and records retrieved, endometriosis diagnosis was confirmed. Furthermore, requests to review medical records were also sent to those women who indicated having a hysterectomy during the time of endometriosis diagnosis. In 79.6\% (144/188) of the records retrieved, a diagnosis of endometriosis at the time of the hysterectomy was confirmed; however, in only $5.5 \%$ (9/163), endometriosis was the primary indication for the surgical procedure. Thus, in order to minimize the magnitude of misclassification of the outcome and confounding by indication for hysterectomy, only incident cases that reported a laparoscopic confirmation of their diagnosis were included in the study. 
Using laparoscopic confirmation to define endometriosis cases results in a complex relation between endometriosis and fertility status. The baseline prevalence of infertility (defined as attempting to conceive for $>12$ months without success) in this cohort is higher among those with laparoscopically-confirmed endometriosis (20\%) compared to those reporting endometriosis without laparoscopic confirmation (4\%). Among women with infertility, many may have only been diagnosed with endometriosis during an infertility evaluation. In contrast, women with endometriosis without infertility are more likely to have pain as an indication for laparoscopic evaluation. Because women with endometriosis with infertility may have a higher prevalence of asymptomatic disease secondary to other primary causes of infertility, the risk factors for endometriosis with infertility may differ from those for endometriosis without infertility. Therefore, we conducted analyses stratified by fertility status.

\section{Assessment of Dietary Exposures and Covariates}

Diet was assessed via the semi-quantitative food frequency questionnaires (FFQ) in 1991 and every four years thereafter. Women were asked to report their usual intake during the past year on more than 130 food items. Each question had nine possible responses, ranging from "never or less than once per month" to "six or more times per day." We calculated the intakes of total and specific types of meat by multiplying the portion size of a single serving of each meat item by its reported frequency of intake. The validity of the questionnaire and of meat intake and related nutrients has been extensively assessed ${ }^{10-12}$. For intake of meats, the correlation coefficients comparing the average of prospectively collected 1-week diet records and the FFQ were 0.67 for chicken without skin, 0.58 for chicken with skin, 0.55 for processed meats, 0.38 for hamburgers, 0.56 for hot dogs, 0.66 for fish, and 0.77 for eggs 12 . For nutrients concentrated in these foods, the correlation coefficients were 0.68 for zinc with supplements, 0.52 zinc from foods alone, 0.71 vitamin B1 with supplements, 0.54 vitamin B1 from foods alone, 0.74 vitamin B12 with supplements, 0.56 vitamin B12 from foods alone, 0.58 iron with supplements, 0.56 iron from foods alone, and palmitic fatty acid 0.70 .

Analyses for meat consumption were conducted using the following categories: red meat including processed and organ meats (hot dogs, bacon, sliced processed meat, hamburger, beef/pork/lamb sandwich, pork, beef, liver), unprocessed red meat (hamburger, beef/pork/ lamb sandwich, pork, beef, liver), processed red meat (hotdog, bacon and sliced processed meat), poultry (chicken, turkey), fish (canned tuna, dark meat fish, other fish), shellfish (shrimp, lobster, scallops), and eggs.

\section{Statistical analyses}

Participants were followed from return of the baseline FFQ (1991) until self-report of laparoscopically confirmed endometriosis, death, diagnosis of cancer (excluding nonmelanoma skin cancer), hysterectomy, menopause, loss to follow-up or June 1, 2013, which ever occurred first. Participants who reported physician-diagnosed endometriosis with no laparoscopic confirmation were censored at the time of that report but were allowed to reenter the analysis population if they reported laparoscopic confirmation on a subsequent questionnaire. Time-varying Cox proportional hazard regression models with age in months and the 2-year questionnaire cycle as the time scales were used to calculate incidence rate 
ratios (RR) and 95\% confidence intervals (95\% CI), using the lowest category of each food or nutrient as the reference. Since the temporal relation between diet and endometriosis is uncertain, we examined timing of dietary exposure in three ways. First, in the baseline diet analysis (1991), we examined only diet reported in 1991 in relation to cases reported throughout the follow-up period (1991-2013). Next, in the simple update analysis, 1991 intake was assigned to the 1991-1995 follow-up period, 1995 intake was assigned to the 1995-1999 follow-period, 1999 intake was assigned to 1999-2003 follow-up period, and so forth. Finally, for cumulative averaged intake analysis, 1991 intake was assigned to followup through 1995, the average of 1991 and 1995 intakes was assigned to the 1995-1999 follow-up period, the average of 1991, 1995, and 1999 intakes was assigned to the 19992003 follow-up period, and so forth. As the results for each approach were similar we present results for only the cumulative averaged models, which minimizes measurement error due to random within-person variability and is representative of long-term intake ${ }^{13}$. We estimated the effect of substituting one meat for another by simultaneously including terms for all meats in the same model and using the differences between regression coeficients as the estimated substitution effect ${ }^{14}$. To explore if the effect of meat intake was explained by nutrient(s) concentrated in these foods we simultaneously included the meat (e.g., red meat) and nutrient (e.g., iron) variables in a regression model.

The multivariable model was adjusted for potential confounders that were chosen a priori due to their association with endometriosis including race (Caucasian vs. women of color), parity (nulliparous, $1,2,3,4+$ pregnancies lasting $>6$ months), BMI $(<19,19-20.4,20.5-$ $\left.21.9,22-24.9,25-29.9,30+\mathrm{kg} / \mathrm{m}^{2}\right)$, length of menstrual cycle between ages $18-22(<26$, $26-31,32-50,51+$ days and irregular), age at menarche ( $<10$ years, $10,11,12,13,14,15$, $15,16,>16)$, pelvic or breast exam in the past year, and total caloric intake. The following covariates were updated throughout the analysis whenever new information was available from the biennial questionnaires: parity, BMI, pelvic/breast exam, and total caloric intake. Oral contraceptive use and cigarette smoking were also evaluated as potential confounders but did not materially influence the effect estimates so were not included in the final models. Tests for linear trend in ordinal categorical exposures were calculated by assigning a median value of each category to all participants in that group.

Since some risk factors for endometriosis may differ by fertility status (defined as attempting to conceive for $>12$ months without success), we examined report of infertility as an effect modifier using likelihood ratio tests comparing models with both the main effects and the cross-product term between the exposure variable and fertility status to those with the main effects only. Statistical analyses were performed using SAS version 9.4 (SAS Institute Inc, Cary, North Carolina).

\section{Results}

We documented 3,800 cases of laparoscopically-confirmed diagnosis of endometriosis during 1,019,294 person-years of follow-up. Based on baseline (1991) characteristics, women with the highest red meat intake were more likely to be overweight or obese, more likely to be current smokers, and to have used oral contraceptives. They were less likely to 
be nulliparous, to have had a recent gynecologic exam and had a greater total caloric intake (Table 1).

Red meat intake was associated with a higher risk of endometriosis (Table 2). Women consuming more than two servings of red meat per day had a 56\% higher risk of laparoscopically-confirmed endometriosis compared to women consuming $\triangleleft$ serving per week (95\% CI=1.22-1.99; $\left.\mathrm{P}_{\text {trend }}<0.0001\right)$. This risk was driven primarily by the association with non-processed red meats but women in the highest category of processed red meat intake also had a significantly higher risk of endometriosis. Non-processed red meat was associated with a $57 \%$ increase in risk $\left(95 \% \mathrm{CI}=1.35-1.83\right.$; $\left.\mathrm{P}_{\text {trend }}<0.0001\right)$ for persons consuming $\geq 2$ servings/day versus $\leq 1$ servings/week while processed red meat was associated with a $20 \%$ increased risk $\left(95 \% \mathrm{CI}=1.06-1.37 ; \mathrm{P}_{\text {trend }}=0.02\right.$ for $\geq 5$ servings/week versus $<1$ serving/month). There was a statistically significant linear trend towards higher risk of endometriosis with increasing intake of poultry $\left(\mathrm{P}_{\text {trend }}=0.03\right)$. However, none of the estimates for specific categories of intake were significant and there was not a clear overall pattern of association. No associations were observed with fish, shellfish, or eggs (Table 2). Substitution analyses suggested that consuming fish, shellfish, or eggs instead of red meat was associated with a lower risk of endometriosis (Figure 1).

The association between red meat intake and endometriosis were modified by fertility status (Table 2). Specifically, the association between red meat and non-processed red meat intake and endometriosis risk was strongest among women who had not reported infertility (RR for red meat $=1.52$ comparing $>2 /$ day versus $\leq 1 /$ week; $95 \% \mathrm{CI}=1.16-1.98 ; \mathrm{P}_{\text {trend }}<0.0001$ and $\mathrm{RR}$ for non-processed red meat $=1.58$ comparing $\geq 2$ /day versus $\unlhd /$ week; $95 \% \mathrm{CI}=1.34$ 1.88; $\left.\mathrm{P}_{\text {trend }}<0.0001\right)$. The corresponding RRs for those reporting infertility was 1.21 for red meat $\left(95 \% \mathrm{CI}=0.64-2.29 ; \mathrm{P}_{\text {trend }}=0.89\right)$ and 1.08 for non-processed red meat $(95 \% \mathrm{CI}=0.74$ $\left.1.57 ; \mathrm{P}_{\text {trend }}=0.57\right)$.

Last, we evaluated whether the association of red meat intake with endometriosis was explained by nutrients highly concentrated in red meats (iron, heme iron, zinc, vitamin B1, vitamin B12, animal fat, and palmitic acid). Intake of heme iron was associated with a significantly increased risk of endometriosis $(\mathrm{RR}=1.30,95 \% \mathrm{CI}=1.17-1.44$, comparing quintile (Q) 5 vs. Q1; $\mathrm{P}_{\text {trend }}<0.0001$ ) (Supplemental Table 1). When red meat and heme iron were simultaneously included in the regression models, the associations for both were attenuated but remained significant $(\mathrm{RR}=1.36,95 \% \mathrm{CI}=1.04-1.77>2$ servings of red meat/day vs. $\unlhd$ serving of red meat/week and $\mathrm{RR}=1.17,95 \% \mathrm{CI}=1.03-1.33$ comparing heme iron Q5 to Q1). Adjustment for zinc, vitamin B1, and vitamin B12 did not materially influence the results while adjustment for animal fat and palmitic acid strengthened the results for red meat (Table 3) but did not appreciably alter the results for other meat categories (data not shown).

\section{Comment}

In this prospective cohort of premenopausal women, we observed that red meat, both processed and non-processed, was associated with an increased risk of laparoscopicallyconfirmed endometriosis. This association was strongest among women who had never 
reported infertility. Heme iron intake was also associated with endometriosis risk and explained part, but not all, of the association between red meat intake and endometriosis.

Our results suggest that higher intake of red meat increases the risk of endometriosis diagnosis in a dose response manner. Red meat consumption has been linked to numerous chronic diseases including diabetes, cardiovascular disease, some cancers and recently, has been linked to total, CVD and cancer mortality ${ }^{15}$. Prior to this study, only two case-control studies had investigated the association between consumption of meat products and endometriosis risk ${ }^{7}$. An Italian case-control study observed a significant increase in endometriosis risk for women in the highest tertile of red meat $(\mathrm{OR}=2.0,95 \% \mathrm{CI}=1.4-2.8$; $\left.\mathrm{P}_{\text {trend }}=0.0004\right)$ and ham $\left(\mathrm{OR}=1.8,95 \% \mathrm{CI}=1.3-2.5, \mathrm{P}_{\text {trend }}=0.001\right)$ consumption compared to those in the lowest ${ }^{7}$. In contrast, a Washington state based case-control study reported no association with red meat intake $\left(\mathrm{RR}=0.9,95 \% \mathrm{CI}=0.6-1.4 ; \mathrm{P}_{\text {trend }}=0.74\right.$ for $>4$ servings/day vs $\mathcal{\Omega}$ servings/day) ${ }^{8}$. Similar to our results, neither study reported an association with fish intake and endometriosis risk.

Although the physiological mechanism of how diet affects endometriosis is not fully understood, it has been hypothesized to involve circulating steroid hormones ${ }^{16}$. Endometriosis may be affected by the animal fat present in meat, which is supported by the positive association previously observed between palmitic acid and endometriosis risk in the NHSII ${ }^{17}$, and evidence that reduction in fat consumption leads to a decrease in endogenous estrogens ${ }^{18,19}$. Red meat may also influence estrogen levels directly as a result of exogenous hormone exposure from sex steroid hormone-treated animals ${ }^{20}$ and epidemiologic studies have demonstrated that the consumption of non-processed red meat is associated with decreased SHBG and increased estradiol concentrations ${ }^{3}$. Increased endogenous estrogen can also stimulate the formation of prostaglandins, inducing the release of aromatase $\mathrm{P} 450$, increasing estrogen and prostaglandin concentrations, and favoring the inflammatory nature of endometriosis ${ }^{6}$. In our sensitivity analyses, we separately controlled for animal fat and palmitic acid and in both models the relative risk estimates for red meat were enhanced, suggesting that increased intake of red meat may have effects independent of palmitic acid or animal fat.

The finding that the effect estimates were attenuated when heme iron was included in the multivariable model may suggest that heme iron itself is associated with an increased endometriosis risk and that increasing intake of red meat is associated with increased endometriosis risk at least partially independent of heme iron. However, since red meat was the largest contributor to heme iron intake in this cohort it is difficult to completely disentangle these two nutritional variables. Heme iron is a form of iron that is present in hemoglobin and myoglobin of animal sources, whereas non-heme iron comes primarily from plants sources. While heme iron contributes to a smaller proportion of overall iron intake, its absorption rate is much higher and thus, it could contribute to as much as $\geq 40 \%$ of total absorbed iron ${ }^{21-23}$. These associations we observed with heme iron are consistent with the inflammatory state and oxidative stress mechanisms that have been proposed as potential contributors to the pathophysiology of the endometriosis ${ }^{24-26}$. It is known that erythrocytes release hemoglobin, and its by-products, iron and heme into the peritoneal cavity as a result of retrograde menstruation, and if iron and heme are not chelated, they form harmful 
reactive oxygen species, a form of oxidative stress. Further, it has been observed that women with endometriosis have lower concentrations of hemoplexin, a major transporter protein for heme iron ${ }^{27}$.

We observed from our study that the associations with non-processed and processed red meat were strongest among women who had not reported infertility. Although in this study we cannot tease apart the influence of dietary factors on disease establishment/progression from the influence of these components on the experience of pain which influences the likelihood of diagnosis, the fact that the associations were not observed in those reporting infertility suggests that the association may be due to an influence on pain symptoms. Therefore, if red meat consumption increases pain symptoms, then this could manifest as a increase in the incidence of endometriosis diagnosis, while the incidence of disease itself may be unaltered. A previous cross-over study in women with dysmenorrhea reported that a low-fat vegetarian diet increased SHBG concentrations and decreased pain ${ }^{28}$. This provides a potential pathway through which red meat, which has been shown to decrease SHBG levels and increase estradiol ${ }^{3}$, might influence pain in women with endometriosis.

As with all studies, limitations need to be considered. The NHSII cohort utilized selfreported information, and thus, outcome misclassification is a possibility but is reduced due restricting our case definition to laparoscopically diagnosed endometriosis which have a high level of validity $(>95 \%)^{29}$. While some women with undiagnosed endometriosis are undoubtedly present in the cohort, the prevalence of endometriosis in an asymptomatic population is estimated to be $<2 \%{ }^{30}$. Thus, among our population of over 81,000 women, any influence of these undiagnosed cases on results is likely minimal. In addition, a limitation of the FFQ is that it can misclassify individuals with unusual diets and may introduce systematic error. This measurement error would likely bias effect estimates towards the null; however, the use of repeated questionnaires minimizes this bias. Nevertheless, the FFQ is useful in measuring average intake over an extended period, which is of interest to most epidemiological studies. Furthermore, comparison of the FFQ and other self-report methods such as repeated 24-hour recalls and repeated 7-day diet records against objective biomarkers of intake suggest that the latter offer no meaningful advantage over the FFQ for assessment of diet in epidemiologic studies ${ }^{10}$. Finally, residual or unmeasured confounding by factors that are associated with dietary habits and laparoscopic diagnosis of endometriosis is a possibility. However, we were able to adjust for lifestyle factors including access to health care (e.g., recency of pelvic/breast exam) as established endometriosis risk factors.

The prospective design of our study is a substantial strength, especially compared to casecontrol designs with dietary exposures due to the potential for recall bias and the influence of disease symptoms on current dietary choices in case-control studies. In addition, unlike some previous studies that used an unvalidated food questionnaire to collect dietary information, which did not allow for quantification and control for total energy intake in their analyses, we were able to adjust for total calories which minimizes correlated errors and decreases between-person variation ${ }^{31}$. This is especially important for diseases such as endometriosis where an association between body size and disease risk has been observed $9,32-34$. 
Our prospective analysis among premenopausal US registered nurses suggest that decreasing red meat intake may be important modifiable risk factor for endometriosis, particularly in regards to women presenting with pain symptoms. Future dietary intervention studies among women with endometriosis could help confirm this observation and provide further insight the differential associations observed by fertility status.

\section{Supplementary Material}

Refer to Web version on PubMed Central for supplementary material.

\section{Acknowledgments}

Role of Funding Support: This work was supported by research grants HD4854, HD52473, and HD57210 from the Eunice Kennedy Shriver National Institute of Child Health and Human Development, and grant P30 DK046200 from the National Institutes of Diabetes and Digestive and Kidney Diseases. The Nurses' Health Study II is supported by the Public Health Service grant UM1 CA176726 from the National Cancer Institute, NIH, U.S. Department of Health and Human Services. HRH is supported by the National Cancer Institute, National Institutes of Health (K22 CA193860).

We are indebted to the participants of the Nurses' Health Study II for their outstanding dedication.

\section{References}

1. Wheeler JM. Epidemiology of endometriosis-associated infertility. The Journal of reproductive medicine. 1989; 34(1):41-46. [PubMed: 2704007]

2. Eskenazi B, Warner ML. Epidemiology of endometriosis. Obstetrics and gynecology clinics of North America. 1997; 24(2):235-258. [PubMed: 9163765]

3. Brinkman MT, Baglietto L, Krishnan K, et al. Consumption of animal products, their nutrient components and postmenopausal circulating steroid hormone concentrations. European journal of clinical nutrition. 2010; 64(2):176-183. [PubMed: 19904296]

4. Bartram HP, Gostner A, Scheppach W, et al. Effects of fish oil on rectal cell proliferation, mucosal fatty acids, and prostaglandin E2 release in healthy subjects. Gastroenterology. 1993; 105(5):13171322. [PubMed: 8224635]

5. Harel Z, Biro FM, Kottenhahn RK, Rosenthal SL. Supplementation with omega-3 polyunsaturated fatty acids in the management of dysmenorrhea in adolescents. American journal of obstetrics and gynecology. 1996; 174(4):1335-1338. [PubMed: 8623866]

6. Seli E, Berkkanoglu M, Arici A. Pathogenesis of endometriosis. Obstetrics and gynecology clinics of North America. 2003; 30(1):41-61. [PubMed: 12699257]

7. Parazzini F, Chiaffarino F, Surace M, et al. Selected food intake and risk of endometriosis. Human reproduction (Oxford, England). 2004; 19(8):1755-1759.

8. Trabert B, Peters U, De Roos AJ, Scholes D, Holt VL. Diet and risk of endometriosis in a population-based case-control study. The British journal of nutrition. 2011; 105(3):459-467. [PubMed: 20875189]

9. Missmer SA, Hankinson SE, Spiegelman D, Barbieri RL, Marshall LM, Hunter DJ. Incidence of laparoscopically confirmed endometriosis by demographic, anthropometric, and lifestyle factors. American journal of epidemiology. 2004; 160(8):784-796. [PubMed: 15466501]

10. Yuan C, Spiegelman D, Rimm EB, et al. Relative Validity of Nutrient Intakes Assessed by Questionnaire, 24-Hour Recalls, and Diet Records Compared With Urinary Recovery and Plasma Concentration Biomarkers: Findings for Women. American journal of epidemiology. 2017

11. Yuan C, Spiegelman D, Rimm EB, et al. Validity of a Dietary Questionnaire Assessed by Comparison With Multiple Weighed Dietary Records or 24-Hour Recalls. American journal of epidemiology. 2017; 185(7):570-584. [PubMed: 28338828] 
12. Salvini S, Hunter DJ, Sampson L, et al. Food-based validation of a dietary questionnaire: the effects of week-to-week variation in food consumption. International journal of epidemiology. 1989; 18(4):858-867. [PubMed: 2621022]

13. Hu FB, Stampfer MJ, Rimm E, et al. Dietary fat and coronary heart disease: a comparison of approaches for adjusting for total energy intake and modeling repeated dietary measurements. American journal of epidemiology. 1999; 149(6):531-540. [PubMed: 10084242]

14. Halton TL, Willett WC, Liu S, Manson JE, Stampfer MJ, Hu FB. Potato and french fry consumption and risk of type 2 diabetes in women. The American journal of clinical nutrition. 2006; 83(2):284-290. [PubMed: 16469985]

15. Pan A, Sun Q, Bernstein AM, et al. Red meat consumption and mortality: results from 2 prospective cohort studies. Archives of internal medicine. 2012; 172(7):555-563. [PubMed: 22412075]

16. Missmer SA, Cramer DW. The epidemiology of endometriosis. Obstetrics and gynecology clinics of North America. 2003; 30(1):1-19. vii. [PubMed: 12699255]

17. Missmer SA, Chavarro JE, Malspeis S, et al. A prospective study of dietary fat consumption and endometriosis risk. Human reproduction (Oxford, England). 2010; 25(6):1528-1535.

18. Prentice R, Thompson D, Clifford C, Gorbach S, Goldin B, Byar D. Dietary fat reduction and plasma estradiol concentration in healthy postmenopausal women. The Women's Health Trial Study Group. Journal of the National Cancer Institute. 1990; 82(2):129-134. [PubMed: 2294222]

19. Wu AH, Pike MC, Stram DO. Meta-analysis: dietary fat intake, serum estrogen levels, and the risk of breast cancer. Journal of the National Cancer Institute. 1999; 91(6):529-534. [PubMed: 10088623]

20. Andersson AM, Skakkebaek NE. Exposure to exogenous estrogens in food: possible impact on human development and health. European journal of endocrinology. 1999; 140(6):477-485. [PubMed: 10366402]

21. Carpenter CE, Mahoney AW. Contributions of heme and nonheme iron to human nutrition. Critical reviews in food science and nutrition. 1992; 31(4):333-367. [PubMed: 1581009]

22. Hunt JR. Moving toward a plant-based diet: are iron and zinc at risk? Nutrition reviews. 2002; 60(5 Pt 1):127-134. [PubMed: 12030275]

23. Hurrell R, Egli I. Iron bioavailability and dietary reference values. The American journal of clinical nutrition. 2010; 91(5):1461s-1467s. [PubMed: 20200263]

24. Donnez J, Binda MM, Donnez O, Dolmans MM. Oxidative stress in the pelvic cavity and its role in the pathogenesis of endometriosis. Fertility and sterility. 2016; 106(5):1011-1017. [PubMed: 27521769]

25. Lousse JC, Van Langendonckt A, Defrere S, Ramos RG, Colette S, Donnez J. Peritoneal endometriosis is an inflammatory disease. Frontiers in bioscience (Elite edition). 2012; 4:23-40. [PubMed: 22201853]

26. Van Langendonckt A, Casanas-Roux F, Donnez J. Oxidative stress and peritoneal endometriosis. Fertility and sterility. 2002; 77(5):861-870. [PubMed: 12009336]

27. Wolfler MM, Meinhold-Heerlein IM, Henkel C, et al. Reduced hemopexin levels in peritoneal fluid of patients with endometriosis. Fertility and sterility. 2013; 100(3):777-781. [PubMed: 23755951]

28. Barnard ND, Scialli AR, Hurlock D, Bertron P. Diet and sex-hormone binding globulin, dysmenorrhea, and premenstrual symptoms. Obstetrics and gynecology. 2000; 95(2):245-250. [PubMed: 10674588]

29. Missmer SA, Hankinson SE, Spiegelman D, Barbieri RL, Michels KB, Hunter DJ. In utero exposures and the incidence of endometriosis. Fertility and sterility. 2004; 82(6):1501-1508. [PubMed: 15589850]

30. Zondervan KT, Cardon LR, Kennedy SH. What makes a good case-control study? Design issues for complex traits such as endometriosis. Human reproduction (Oxford, England). 2002; 17(6): 1415-1423.

31. WillettW. Nutritional Epidemiology2. New York, New York: Oxford University, Inc; 1998

32. Ferrero S, Anserini P, Remorgida V, Ragni N. Body mass index in endometriosis. European journal of obstetrics, gynecology, and reproductive biology. 2005; 121(1):94-98. 
33. Matalliotakis IM, Cakmak H, Fragouli YG, Goumenou AG, Mahutte NG, Arici A. Epidemiological characteristics in women with and without endometriosis in the Yale series. Archives of gynecology and obstetrics. 2008; 277(5):389-393. [PubMed: 17922285]

34. Vitonis AF, Baer HJ, Hankinson SE, Laufer MR, Missmer SA. A prospective study of body size during childhood and early adulthood and the incidence of endometriosis. Human reproduction (Oxford, England). 2010; 25(5):1325-1334. 


\section{Implications and Contributions}

Only two case-control studies have examined the associations between consumption of meat products and endometriosis risk with inconsistent results. Thus we sought to examine the association between intake of red meat, poultry, fish, and seafood and risk of laparoscopically-confirmed endometriosis in a prospective setting. We observed that red meat, both processed and non-processed, was associated with an increased risk of laparoscopically-confirmed endometriosis and this association was strongest among women who had never reported infertility. The stronger association among women who had not reported infertility suggests that the association may be due to an influence of diet on pain symptoms. 


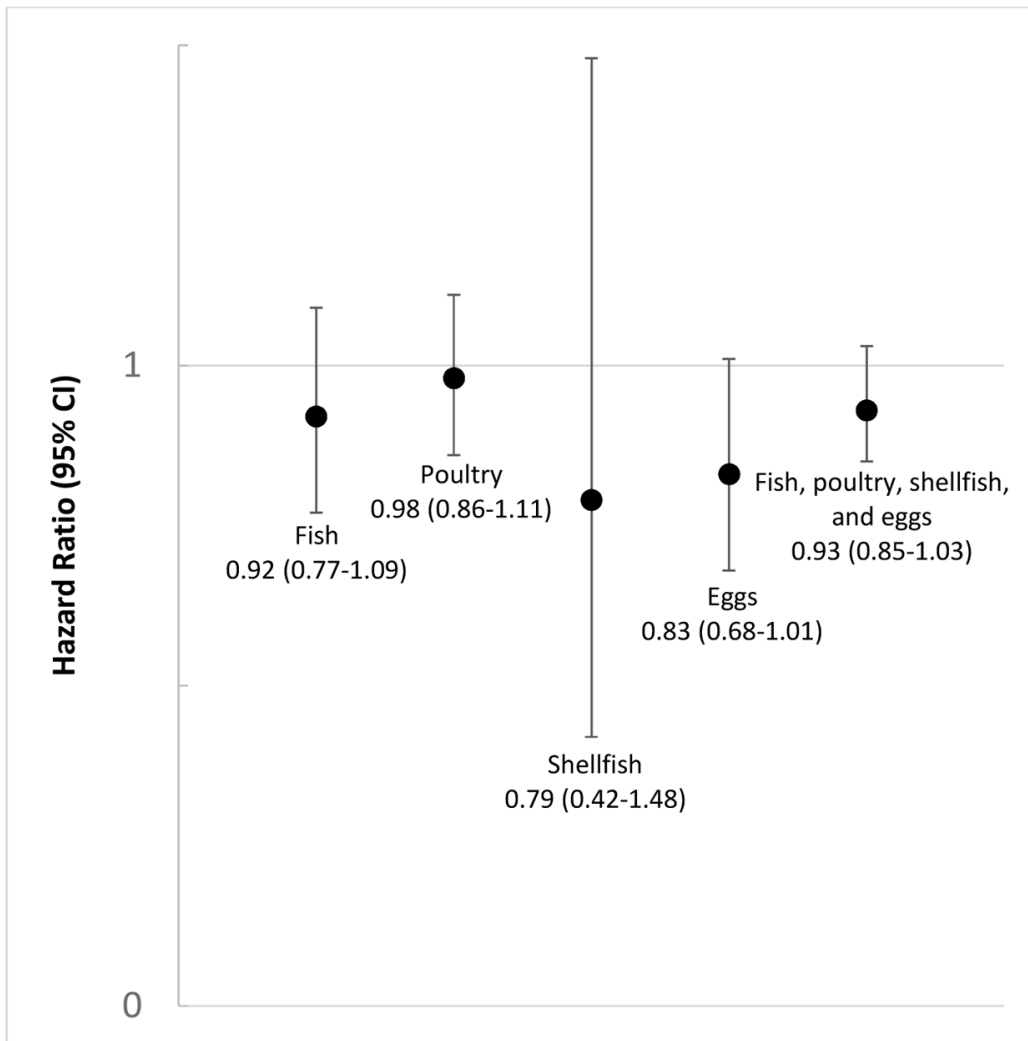

Figure 1.

Risk of endometriosis associated with replacement of 1 serving/day of red meat with other animal protein sources 


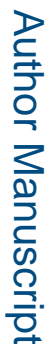

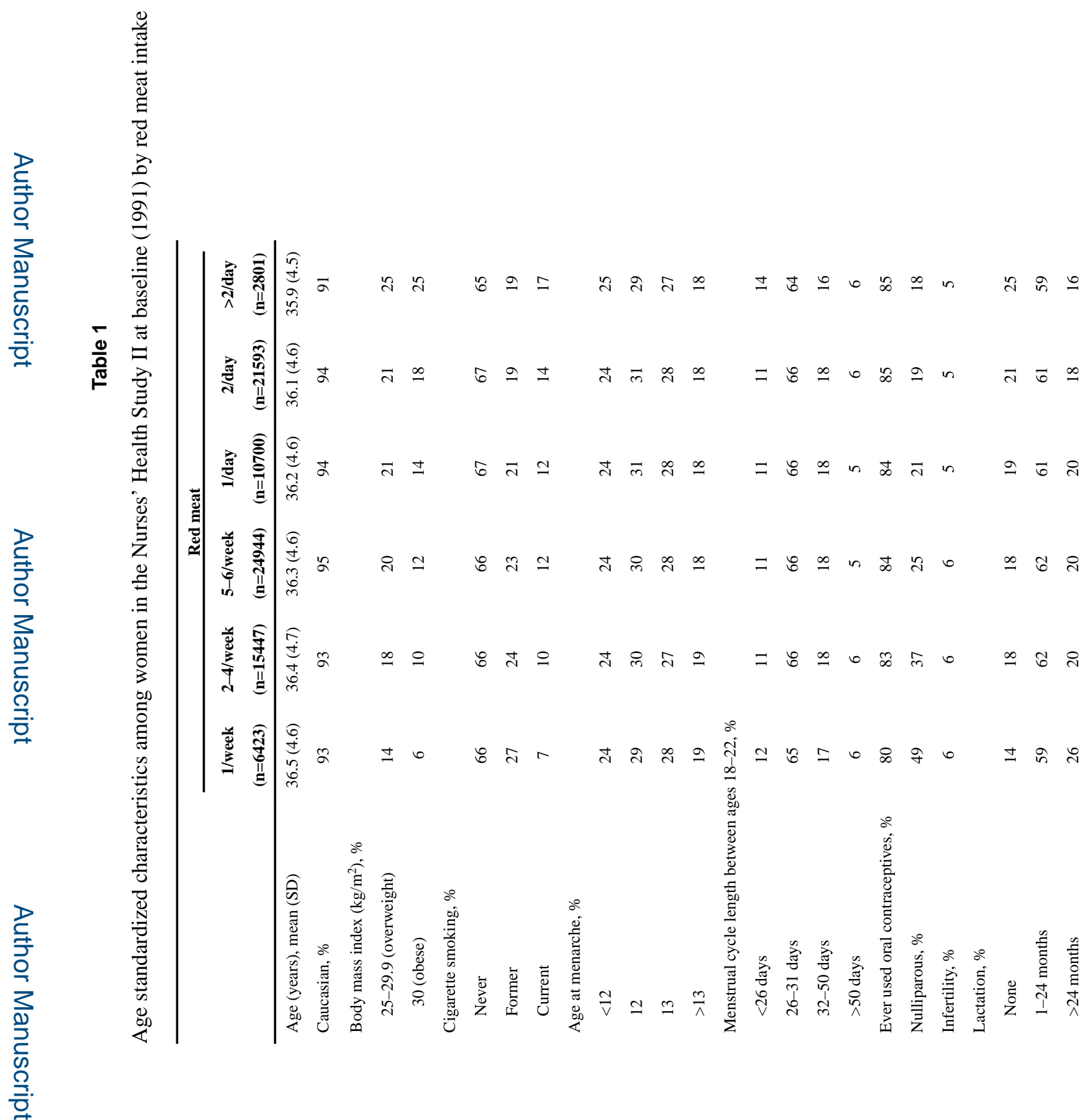

Am J Obstet Gynecol. Author manuscript; available in PMC 2019 August 01. 


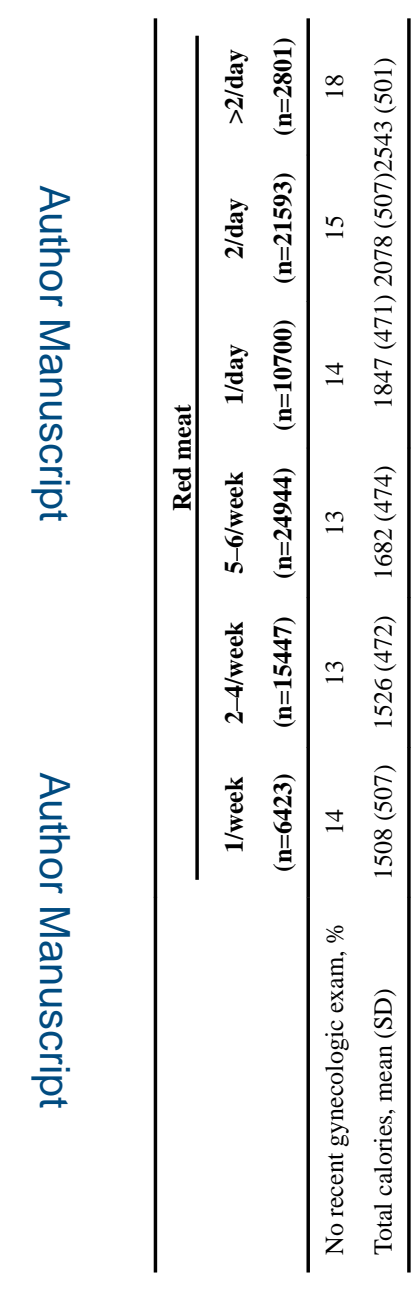

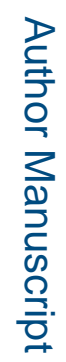

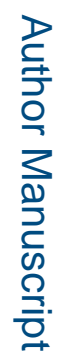




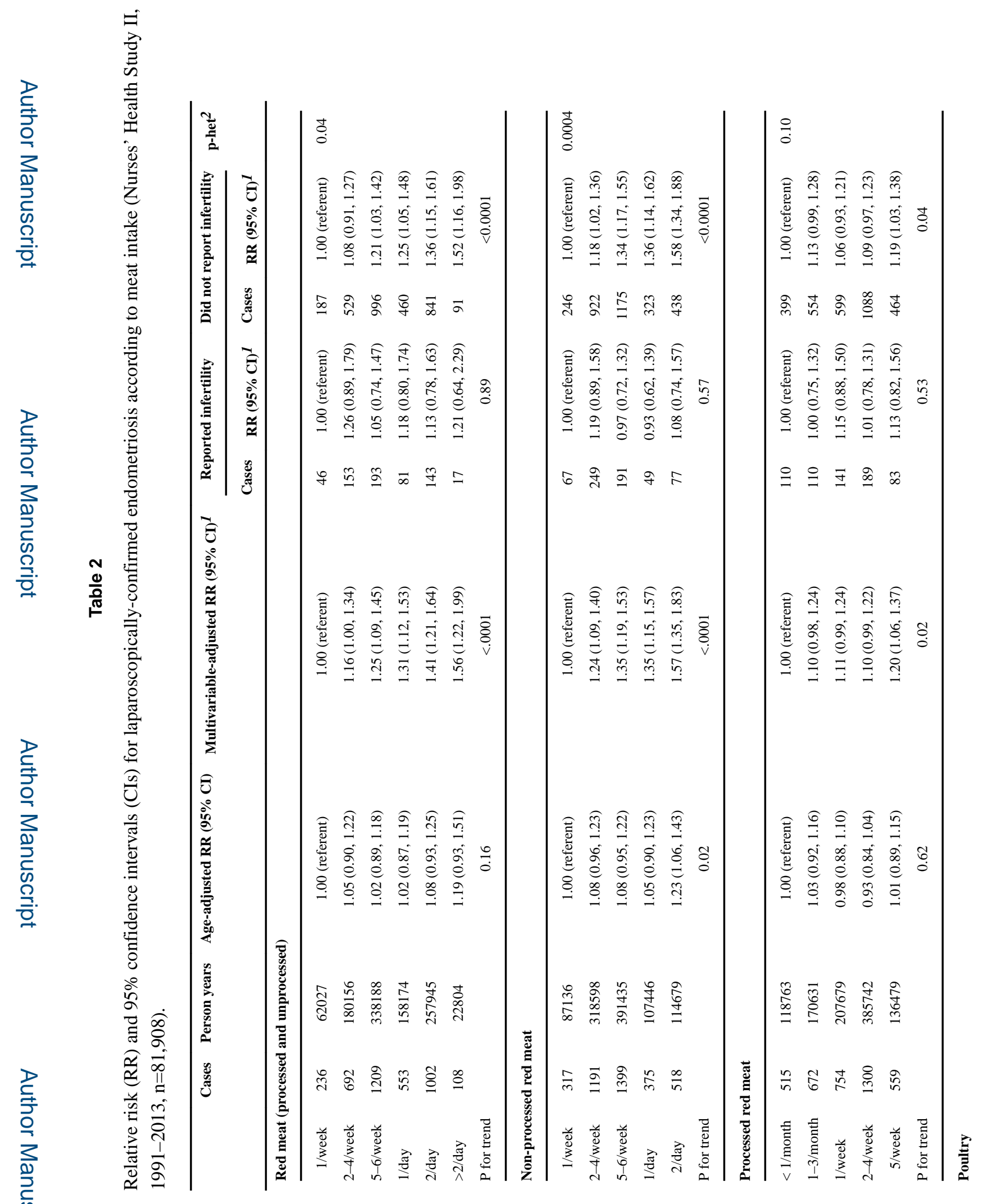


YAMAMOTO et al.

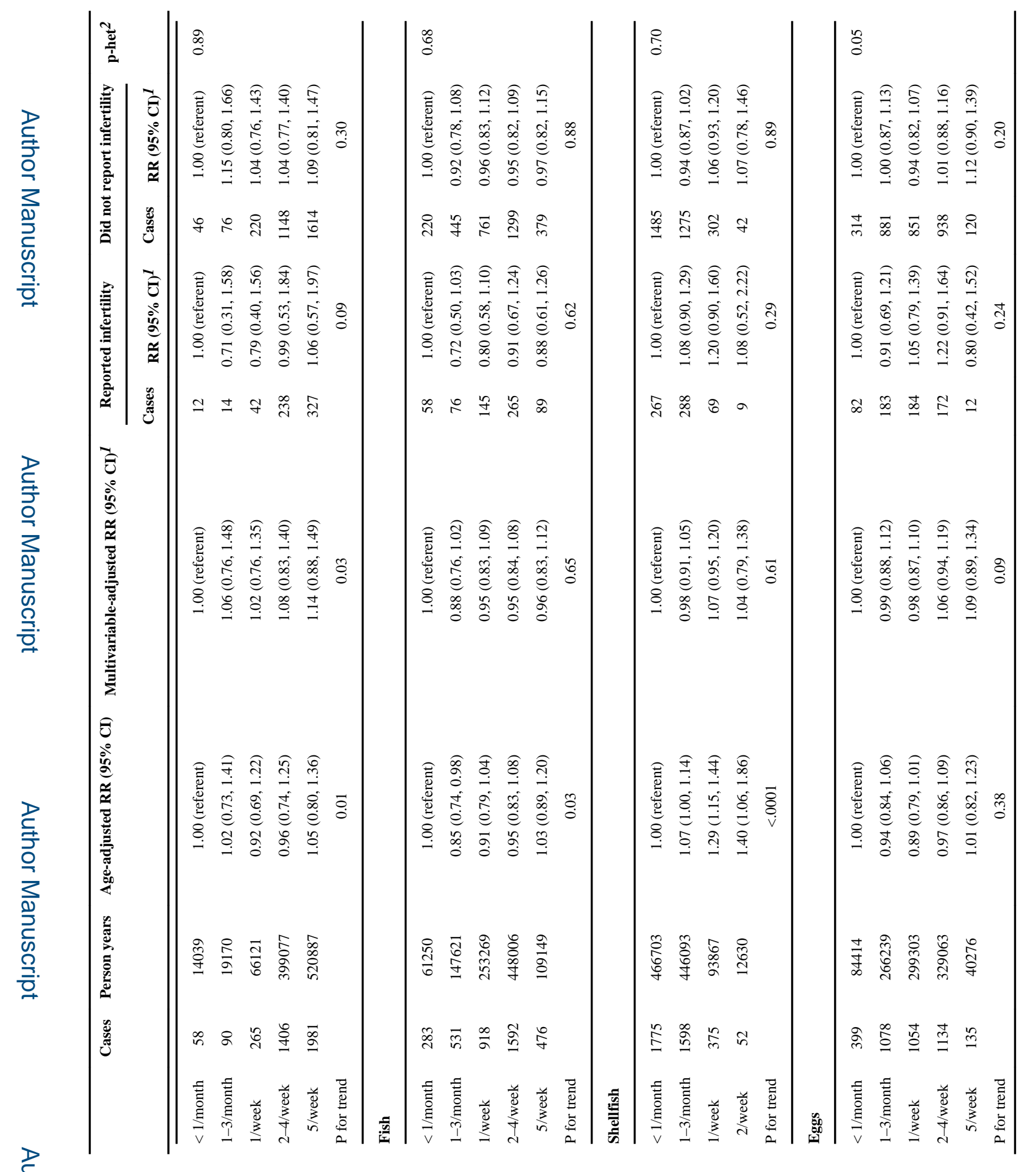

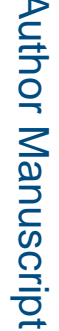




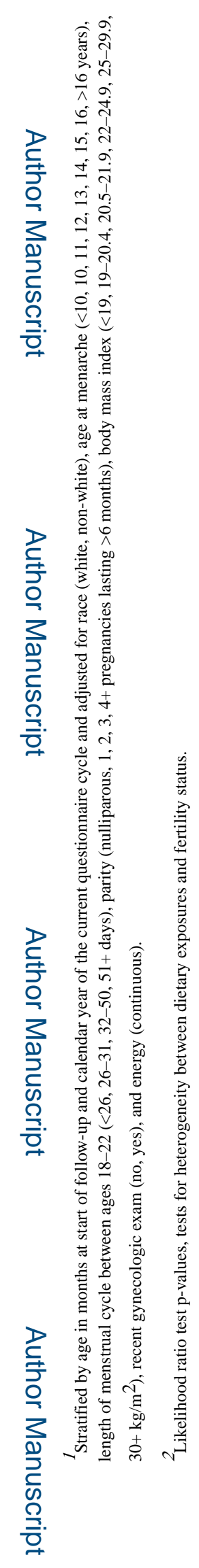




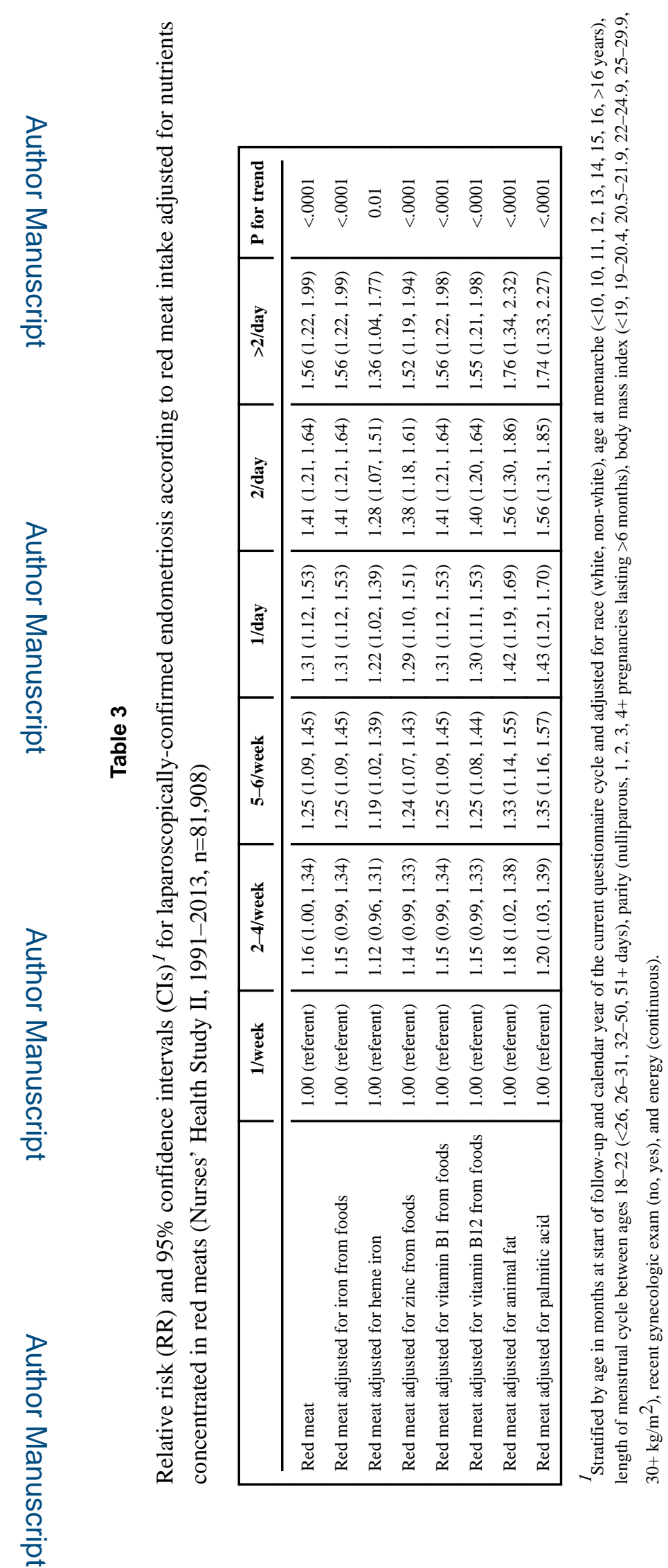

Am J Obstet Gynecol. Author manuscript; available in PMC 2019 August 01. 